\title{
Risk Aversion in Finite Markov Decision Processes Using Total Cost Criteria and Average Value at Risk
}

\author{
Stefano Carpin
}

\author{
Yin-Lam Chow
}

\author{
Marco Pavone
}

recently found applications in domains such as robotics [19]. The term risk aversion refers to a preference for stochastic realizations with limited deviation from the expected value. In risk averse optimal control one may prefer a policy with higher cost in expectation but lower deviations to one with lower cost but possibly larger deviations. Particularly in the context of robotic planning, introducing risk aversion in MDPs is crucial to guarantee mission safety. However introducing risk aversion in MDPs creates a number of additional theoretical and computational hurdles. For example, in risk averse MDPs, optimal policies are not guaranteed to be Markov stationary but are instead history dependent.

Average Value at Risk (AVaR - also known as Conditional Value at Risk or CVaR) is a risk metric that has gained notable popularity in the area of risk averse control [2], [17]. For a given random value and a predetermined confidence level, the AVaR is the tail average of the distribution exceeding a given confidence level (see Section III for a formal definition). Risk averse policies considering the AVaR metric have been studied for the case of MDPs with finite horizon and discounted infinite horizon cost criteria. In this paper we instead consider how the AVaR metric can be applied when an undiscounted, total cost criterion is considered. In fact, such cost criterion appears particularly useful and natural for robotic applications, whereby one is usually interested in optimizing the undiscounted, total cost accrued during a mission until a random, mission-dependent stopping time. (As an aside, the total cost criterion is the typical cost model for stochastic shortest path problems, see, e.g., [20], [21].) The contribution of this paper is three-fold:

- We identify conditions for the underlying MDP ensuring that the AVaR MDP problem is well defined when the total cost criterion is used.

- We define a surrogate MDP problem that can be efficiently solved and whose solution approximates the optimal policy for the original problem with arbitrary precision.

- We validate our findings on a rapid robotic deployment task where the objective is to maximize the mission success rate under a given temporal deadline [6], [8].

The rest of the paper is organized as follows. We discuss related work in Section $\Pi$ II and provide some background about risk metrics and MDPs in Section III In Section IV we formulate the risk-averse, total cost MDP problem we wish to solve. In Section $\mathrm{V}$ we propose and analyze an approximation strategy for the problem, and in Section VI we provide an algorithmic solution. Simulation results for a rapid deployment problem are given in Section VII and conclusions and future work are discussed in Section VIII. 


\section{RELATED WORK}

For a general introduction to MDPs the reader is referred to textbooks such as [4] or more recent collections such as [9]. As pointed out in the introduction, risk aversion in MDPs has been studied for over four decades, with earlier efforts focusing on exponential utility [12], meanvariance [24], and percentile risk criteria [10]. With regard to mean-variance optimization in MDPs, it was recently shown that computing an optimal policy under a variance constraint is NP-hard [15]. Recently, average value at risk was introduced in [17] in order to model the tail risk of a random outcome and to address some key limitations of the prevailing value-at-risk metric. Efficient methods to compute AVaR are discussed in [18]. Leveraging the recent strides in AVaR risk modeling, there have been a number of efforts aimed at embedding the AVaR risk metric into risk-sensitive MDPs. In [3] the authors address the problem of minimizing the AVaR of the discounted cost over a finite and an infinite horizon, and propose a dynamic programming approach based on state augmentation. Similar techniques can be found in [5], where the authors propose a dynamic programming algorithm for finite-horizon, AVaR-constrained MDPs. The algorithm is proven to asymptotically converge to an optimal risk-constrained policy. However, the algorithm involves computing integrals over continuous variables (Algorithm 1 in [5]) and, in general, its implementation appears quite challenging. A different approach is taken by [7], [16], [25] where the authors consider a finite dimensional parameterization of the control policies, and show that an AVaR MDP can be optimized to a local optimum using stochastic gradient descent (policy gradient). However this approach imposes additional restrictions to the policy space and in general policy gradient algorithms only converge to a local optimum. Haskell and Jain recently considered the problem of risk aversion in MDPs using a framework based on occupancy measures [13] (closely connected to our recent works where constrained MDPs are used to solve the multirobot rapid deployment problem [6], [8]). While their findings are only valid for the case where an infinite horizon discounted cost criterion is considered, the solution we propose uses some of the ideas introduced in [13].

\section{Preliminaries}

In this section we summarize some known concepts about risk metrics and MDPs. The reader is referred to the aforementioned references for more details.

\section{A. Risk}

Consider a probability space $\mathcal{S}=(\Omega, \mathcal{F}, \mathbb{P})$, and let $L^{\infty}$ be the space of all essentially bounded random variables on $\mathcal{S}$. A risk function (or risk metric) $\Gamma: L^{\infty} \rightarrow \mathbb{R}$ is a function that maps an uncertain outcome $Y \in L^{\infty}$ onto the real line $\mathbb{R}$. A risk function that is particularly popular in many financial applications is the value at risk. For $\tau \in(0,1)$ the value at risk of $Y \in L^{\infty}$ at level $\tau$ is defined as

$$
\operatorname{VaR}_{\tau}(Y):=\inf \{\eta \in \mathbb{R}: \operatorname{Pr}(Y \leq \eta) \geq \tau\} .
$$

Here $\operatorname{VaR}_{\tau}(Y)$ represents the percentile value of outcome $Y$ at confidence level $\tau$. Despite its popularity, $\operatorname{VaR}_{\tau}$ has a number of limitations. In particular, VaR is not a coherent risk measure [2] and thus suffers from being unstable (high fluctuations under perturbations) when $Y$ is not normally distributed. More importantly it does not quantify the losses that might be incurred beyond its value in the $\tau$-tail of the distribution [17]. An alternative measure that overcomes most shortcomings of $\mathrm{VaR}$ is the average value at risk, defined as

$$
\operatorname{AVaR}_{\tau}(Y):=\frac{1}{1-\tau} \int_{\tau}^{1} \operatorname{VaR}_{t}(Y) d t,
$$

where $\tau \in(0,1)$ is the confidence level as before. Intuitively, $\mathrm{AVaR}_{\tau}$ is the expectation of $Y$ in the conditional distribution of its upper $\tau$-tail. For this reason, it can be interpreted as a metric of "how bad is bad." $\mathrm{AVaR}_{\tau}$ can be equivalently written as [18]

$$
\operatorname{AVaR}_{\tau}(Y)=\min _{s \in \mathbb{R}}\left\{s+\frac{1}{1-\tau} \mathbb{E}\left[(Y-s)^{+}\right]\right\},
$$

where $x^{+}:=\max (x, 0)$. This paper relies extensively on Eq. (1) and aims at devising efficient methods to approximate the expectation in Eq. (1) when the random variable $Y$ is the total cost of an MDP.

Furthermore, it has been recently shown in [23] that optimizing the CVaR of total reward is equivalent to optimizing the worst-case (robust) expected total reward of a system whose model uncertainty is subjected to a trajectory budget. This finding corroborates the fact that a CVaR risk metric models both the variability of random costs, as well as the robustness to system transition errors.

\section{B. Total Cost, Transient Markov Decision Processes}

For a finite set $S$, let $\mathbb{P}(S)$ indicate the set of mass distributions with support on $S$. A finite, discrete-time Markov Decision Process (MDP) is a tuple $\mathcal{M}=(X, U, \operatorname{Pr}, c)$ where

- $X$, the state space, is a finite set comprising $n$ elements.

- $U$, the control space, is a collection of $n$ finite sets $\left\{U\left(x_{i}\right)\right\}_{i=1}^{n}$. Set $U\left(x_{i}\right), i=1, \ldots, n$, represents the actions that can be applied when in state $x_{i} \in X$. The set of allowable state/action pairs is defined as

$$
\mathcal{K}:=\{(x, u) \in X \times U \mid u \in U(x)\} .
$$

- $\operatorname{Pr}(y \mid x, u): \mathcal{K} \rightarrow \mathbb{R}$ is the transition probability from state $x$ to state $y$ when action $u \in U(x)$ is applied. According to our definitions, $\operatorname{Pr}(\cdot \mid x, u) \in \mathbb{P}(X)$.

- $c: \mathcal{K} \rightarrow \mathbb{R}_{>0}$ is a non-negative cost function. Specifically, $c(x, u)$ is the cost incurred when executing action $u \in U(x)$ at state $x$.

Let $\bar{K}:=\max _{(x, u) \in \mathcal{K}} c(x, u)$ and note that the maximum is attained as $\mathcal{K}$ is a finite set. Define the set $\mathcal{H}_{t}$ of admissible histories up to time $t$ by $\mathcal{H}_{t}:=\mathcal{K} \times \mathcal{H}_{t-1}$, for $t \geq 1$, and $\mathcal{H}_{0}:=X$. An element of $\mathcal{H}_{t}$ has the form $x_{0}, u_{0}, x_{1}, \ldots, x_{t-1}, u_{t-1}, x_{t}$, and records all states traversed and actions taken up to time $t$. In the most general case a policy is a function $\pi: \mathcal{H}_{t} \rightarrow \mathbb{P}\left(U\left(x_{t}\right)\right)$, i.e., it decides which action to take in state $x_{t}$ considering the entire stateaction history. Note that according to this definition a policy is in general randomized. Let $\Pi$ be the set of all policies, i.e., including history-dependent, randomized policies. It is well known that in the standard MDP setting where an expected cost is minimized there is no loss of optimality 
in restricting the optimization over deterministic, stationary Markovian policies, i.e., policies of the type $\pi: X \rightarrow U$. However, in the risk-averse setting one needs to consider the more general class of history-dependent policies [1]. This is achieved through a state augmentation process described later.

Following [13], we define the countable space $(\Omega, \mathbb{B}):=$ $\left(\mathcal{K}^{\infty}, \mathbb{B}\left(\mathcal{K}^{\infty}\right)\right)$, where $\mathcal{K}^{\infty}=\mathcal{K} \times \mathcal{K} \times \mathcal{K} \times \cdots$, is the sample space and $\mathbb{B}\left(\mathcal{K}^{\infty}\right)$ is the Borel field on $\mathcal{K}^{\infty}$. Specific trajectories in the MDP are written as $\omega \in \Omega$, and we denote by $x_{t}(\omega)$ and $u_{t}(\omega)$ the state and actions at time $t$ along trajectory $\omega$. In general the exact initial state $x_{0}$ is unknown. Rather it is described by an initial mass distribution $\beta$ over $X$, i.e., $\beta \in \mathbb{P}(X)$. A policy $\pi$ and initial distribution $\beta$ induce a probability distribution over $(\Omega, \mathbb{B})$, that we will indicate as $\operatorname{Pr}_{\beta}^{\pi}$.

In this paper we focus on transient total cost MDPs, defined as follows. Consider a partition of $X$ into sets $X^{T}$ and $M$, i.e., $X=X^{T} \cup M$ and $X^{T} \cap M=\emptyset$. A transient MDP is an MDP where each policy $\pi$ satisfies the following two properties:

- $\sum_{t=0}^{\infty} \operatorname{Pr}_{\beta}^{\pi}\left[x_{t}=x\right]<\infty$ for each $x \in X^{T}$, i.e., the state will eventually enter set $M$, and

- $P(y \mid x, u)=0$ for each $x \in M, y \in X^{T}, u \in U(x)$, i.e., once the state enters $M$ it cannot leave it.

A transient, total cost MDP is a transient MDP where

- $c(x, u)=0$ for each $x \in M$, i.e., once the state enters $M$ no additional cost is incurred,

- the cost associated with each trajectory $\omega$ is given by

$$
c(\omega):=\sum_{t=0}^{\infty} c\left(x_{t}(\omega), u_{t}(\omega)\right) .
$$

Note that the cost $c(\omega)$ is a random variable depending on both the policy $\pi$ and the initial distribution $\beta$. The name total cost stems from the fact that an (undiscounted) cost is incurred throughout the "lifetime" of the system (i.e., until the state hits the absorbing set).

Transient, total cost MDPs (closely related to stochastic shortest path problems, e.g., [20], [21]) represent an alternative to the more commonly used discounted, infinitehorizon MDPs or finite horizon MDPs. As outlined in the introduction, for many robotic applications the total cost, i.e., $c(\omega)$, is the most appropriate cost function. We justify this statement by noting that most robotic tasks have finite duration but such duration is usually not known in advance. In these circumstances the finite horizon cost is inappropriate because one cannot define the length of the finite horizon up front. Similarly, the discounted infinite horizon cost is also ill suited because the task does not continue forever and the cost will not be exponentially discounted over time.

Without loss of generality, we assume that set $M$ consists of a single absorbing state $x_{M}$ equipped with a single action $u_{x_{M}}$, i.e., $M=\left\{x_{M}\right\}$ and $U\left(x_{M}\right)=u_{x_{M}}$ with $\operatorname{Pr}\left(x_{M} \mid x_{M}, u_{x_{M}}\right)=1$. In the following, with a slight abuse of notation, we denote by $\mathcal{K}$ the set $\left\{(x, u) \in X^{T} \times\right.$ $U \mid u \in U(x)\}$, i.e., we exclude the absorbing state from the definition of $\mathcal{K}$. Moreover, we assume that for a transient, total cost MDP $\beta\left(x_{M}\right)=0$, i.e., the probability of starting at the absorbing state is zero. In fact, whenever $x_{0}=x_{M}$ the resulting state trajectory will deterministically remain in $x_{M}$, and the corresponding cost is zero.

\section{Problem Formulation}

Our problem formulation relies on the following two technical assumptions necessary to establish an a-priori upper bound on the total cost incurred by any trajectory obtained under any policy, and to define an approximate problem that can be efficiently solved.

The first assumption simply requires that all costs in the transient states are positive (recall that we excluded $x_{M}$ when re-defining $\mathcal{K}$.) As it will be shown later, this assumption ensures a non-zero discretization step when approximating the cumulative cost accrued by a system throughout the trajectory $\omega$ until it is absorbed in $x_{M}$.

Assumption 1 (Positivity of costs): All costs in $\mathcal{M}$ except for state $x_{M}$ are positive and bounded, i.e., $\underline{K}:=$ $\min _{(x, u) \in \mathcal{K}} c(x, u)>0$.

When considering cost criteria like finite horizon or discounted infinite horizon with a finite state space, an apriori upper bound on the accrued cost can be immediately established assuming that all costs are finite (a fact crucially exploited in [13]). However, the situation is more complex when considering the total cost case, because without introducing further hypotheses on the structure of the MDP a malicious adversary could establish an history-dependent policy capable of invalidating any a-priori established bound on the $\cos 1$. The second assumption then adds a "global reachability structure" to the MDP problem. To this end, in the following, it will be useful to consider the Markov Chain generated by the MDP when an input is selected for each state. For an MDP $\mathcal{M}$, select $u_{1} \in U\left(x_{1}\right), \ldots, u_{n} \in U\left(x_{n}\right)$. The selected inputs and the transition probabilities in $\mathcal{M}$ define a finite Markov Chain that we indicate as $\mathcal{M C}_{u_{1}, \ldots, u_{n}}$. The state space of $\mathcal{M C}_{u_{1}, \ldots, u_{n}}$ is equal to $X$ and for two states $x_{i}, x_{j} \in X$ the transition probability $\operatorname{Pr}_{i, j}$ is defined as $\operatorname{Pr}_{i, j}=\operatorname{Pr}\left(x_{j} \mid x_{i}, u_{i}\right)$ where $u_{i} \in U\left(x_{i}\right)$ is the input selected in the definition of $\mathcal{M C}_{u_{1}, \ldots, u_{n}}$ and $\operatorname{Pr}$ is the transition probability of the associated MDP.

Assumption 2 (Reachability of $M D P$ ): Let $\mathcal{M C}_{u_{1}, \ldots, u_{n}}$ be the Markov chain induced by the $n$ inputs $u_{i} \in U\left(x_{i}\right)$. Then the absorbing state $x_{M}$, under Markov chain $\mathcal{M C}_{u_{1}, \ldots, u_{n}}$, is reachable from any state $x \in X^{T}$, for all $u_{1} \in U\left(x_{1}\right), \ldots, u_{n} \in U\left(x_{n}\right)$.

We recall that a state $j$ in a Markov chain is said reachable from another state $i$ if there exists an integer $k \geq 1$ such that the probability that the chain will be in state $j$ after $k$ transitions is positive [11]. Note that when Assumption 2 holds, under every policy there is a path of non-zero probability connecting every state to $x_{M}$. Therefore, it is impossible to devise a policy that prevents sure absorption for an arbitrary number of steps. This holds for all policies, including history dependent policies (see Figure 11).

Building upon the previous material, we can now define the problem we aim to solve in this paper:

\footnotetext{
${ }^{1}$ First note that we are seeking a uniform upper bound for all possible policies, including history dependent policies. Hence, given a tentative bound $B$, in the general case one could devise a history dependent policy ensuring that every trajectory generated by the policy is not absorbed in $x_{M}$ in less than $B / \underline{K}$ steps, thus invalidating the bound.
} 


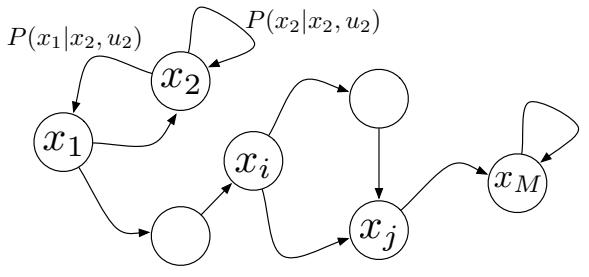

Fig. 1: Meaning of Assumption 2, after one input has been chosen for every state, an associated Markov Chain $\mathcal{M C}_{u_{1}, \ldots, u_{n}}$ is defined. In this Markov Chain, the absorbing state $x_{M}$ is reachable from every state, i.e., at every state there is a path of non-zero probability to $x_{M}$. The probability of a path is given by the product of the probabilities of its edges, i.e., the probability of the path $x_{i} \rightarrow x_{j} \rightarrow x_{M}$ is $\operatorname{Pr}\left(x_{j} \mid x_{i}, u_{i}\right) \operatorname{Pr}\left(x_{M} \mid x_{j}, u_{j}\right)$. This requirement is imposed for every possible choice of the inputs and every policy.

Risk-Averse, total cost MDP - Given a transient total cost MDP satisfying Assumptions 1, 2, and an initial distribution $\beta$, determine a policy $\pi$ that minimizes $\operatorname{AVaR}_{\tau}(c(\omega))$, i.e., find

$$
\pi^{*} \in \arg \min _{\pi \in \Pi} \operatorname{AVaR}_{\tau}(c(\omega)) .
$$

Note that, under the assumption of transient total cost MDP, one can easily verify that $\mathbb{E}[c(\omega)]<\infty$. Since, by equation (1), $\operatorname{AVaR}_{\tau}(c(\omega)) \leq 1 /(1-\tau) \mathbb{E}[c(\omega)]$, one obtains $\operatorname{AVaR}_{\tau}(c(\omega))<\infty$ for all $\omega$, as well. However, to derive an optimization algorithm for the computation of $\pi^{*}$ it is necessary to formulate an a-priori upper bound for the optimal cost in (2). Assumptions 1 and 2 are introduced to ensure that such bound exists and can be computed.

\section{Approximation Strategy}

In this section we study an approximation strategy for the risk averse total cost MDP in equation (2). Similar to the method presented in [13], we aim at solving the problem by using the concept of occupation measures. However, unlike for the cases studied in [13], in total cost MDPs an explicit upper bound for the accrued cost is not available, which makes the solution strategy in [13] not applicable. Our strategy is to find a surrogate to problem (2). By imposing an effective horizon, we construct a total cost MDP with time-out and recast this problem into a bilinear programming problem. Furthermore we characterize the sub-optimality gap for such surrogate approximation. We start with a technical result characterizing the convergence rate to the absorbing state.

\section{A. Convergence rate to the absorbing state}

Consider a selection of inputs $u_{1} \in U\left(x_{1}\right), \ldots, u_{n} \in$ $U\left(x_{n}\right)$ and the corresponding Markov chain $\mathcal{M C}_{u_{1}, \ldots, u_{n}}$. For each state $x \in X^{T}$, let $\operatorname{MinimumPath}_{x \rightarrow x_{M}}\left(\mathcal{M C}_{u_{1}, \ldots, u_{n}}\right)$ denote the simple (i.e., without cycles) path from $x$ to $x_{M}$ of lowest, strictly positive probability. Note that MinimumPath $_{x \rightarrow x_{M}}\left(\mathcal{M C}_{u_{1}, \ldots, u_{n}}\right)$ exists due to Assumption 2. Let $\operatorname{Pr}\left(\operatorname{MinimumPath}_{x \rightarrow x_{M}}\left(\mathcal{M C}_{u_{1}, \ldots, u_{n}}\right)\right)$ be the probability of the path, i.e., the product of the probabilities of all the transitions along the path. Since there are $n$ nodes and by definition the path is simple,
MinimumPath $_{x \rightarrow x_{M}}\left(\mathcal{M C}_{u_{1}, \ldots, u_{n}}\right)$ includes at most $n-1$ transitions between $n$ nodes. Let

$$
\gamma:=\min _{\substack{u_{k} \in U\left(x_{k}\right), k=1 \ldots, n}} \min _{x \in x^{T}} \operatorname{Pr}\left(\operatorname{MinimumPath}_{x \rightarrow x_{M}}\left(\mathcal{M C}_{u_{1}, \ldots, u_{n}}\right)\right) .
$$

Note that the minimum is achieved as the minimization is over a finite set, and that $\gamma$ is strictly positive due to Assumption 2. The constant $\gamma$ lower bounds the probability that, under any policy $\pi \in \Pi$, the absorbing state is reached in no more than $n$ steps, from any state $x \in X^{T}$. We are now in a position to characterize the convergence rate to the absorbing state.

Lemma 1 (Number of stages to reach the absorbing set): For any policy $\pi \in \Pi$ and initial distribution $\beta$,

$$
\operatorname{Pr}_{\beta}^{\pi}\left[x_{k n} \neq x_{M}\right] \leq(1-\gamma)^{k}, \forall k \in \mathbb{N} .
$$

Proof: The claim is proven by induction on $k$. Base case: we prove that

$$
\operatorname{Pr}_{\beta}^{\pi}\left[x_{n} \neq x_{M}\right] \leq 1-\gamma .
$$

Indeed, $\operatorname{Pr}_{\beta}^{\pi}\left[x_{n} \neq x_{M}\right]=\sum_{x \in X_{T}} \operatorname{Pr}_{\beta}^{\pi}\left[x_{n} \neq x_{M} \mid x_{0}=\right.$ $x] \operatorname{Pr}_{\beta}^{\pi}\left[x_{0}=x\right]$. Because of Assumption 11, for any policy $\pi, \operatorname{Pr}_{\beta}^{\pi}\left[x_{n}=x_{M}\right] \geq \gamma$, and the base case follows.

For the inductive step, assume that $\operatorname{Pr}_{\beta}^{\pi}\left[x_{k n} \neq x_{M}\right]<$ $(1-\gamma)^{k}$, for some $k>1$. Then, $\operatorname{Pr}_{\beta}^{\pi}\left[x_{(k+1) n} \neq x_{M}\right]=$ $\operatorname{Pr}_{\beta}^{\pi}\left[x_{(k+1) n} \neq x_{M} \mid x_{k n} \neq x_{M}\right] \operatorname{Pr}_{\beta}^{\pi}\left[x_{k n} \neq x_{M}\right]$. Вy definition of $\gamma$,

$$
\operatorname{Pr}_{\beta}^{\pi}\left[x_{(k+1) n} \neq x_{M} \mid x_{k n} \neq x_{M}\right] \leq(1-\gamma)^{k+1},
$$

and the claim follows.

\section{B. Surrogate problem and approximation bounds}

Our solution strategy is to solve a surrogate problem, whereby after a deterministic number $d \in \mathbb{N}$ of steps, the state moves to the absorbing state $x_{M}$ surely. In other words, $d$ acts as a "timeout" for the MDP problem. The surrogate problem is simpler to solve, and we will show in the following that its solution can approximate the solution of the original problem with arbitrary precision ${ }^{2}$ Denote by $c^{[d]}(\omega)$ the total cost for such surrogate problem. Additionally, for the original problem, let $t^{*}(\omega)$ denote the absorbing time, i.e., the time at which the state reaches $x_{M}$. If $t^{*}(\omega) \leq d$, then the two processes coincide and then $c^{[d]}(\omega)=c(\omega)$. Otherwise, for each trajectory $\omega$ such that $t^{*}(\omega)>d$, the random process is stopped after $d$ steps, and the state goes, deterministically, to $x_{M}$ at stage $d+1$. In such a case one has $c^{[d]}(\omega) \leq c(\omega)$.

We want to characterize the relation between $\operatorname{AVaR}_{\tau}\left(c^{[d]}(\omega)\right)$ (i.e., the risk for the surrogate problem) and $\mathrm{AVaR}_{\tau}(c(\omega))$ (i.e., the risk for the original problem). To this end, let $c_{d}(\omega)$ be the total cost for the original problem up to time $d$, i.e.,

$$
c_{d}(\omega):=\sum_{t=0}^{d} c\left(x_{t}(\omega), u_{t}(\omega)\right) .
$$

\footnotetext{
${ }^{2}$ An alternative strategy would be to investigate reductions of problem (2) to an equivalent risk-averse, discounted, infinite-horizon problem by using, e.g., the results recently presented in [22], and then apply the approach in [13] to the reformulated problem. This is an interesting direction left for future research.
} 
The following lemma shows the equivalence between $c^{[d]}(\omega)$ and $c_{d}(\omega)$.

Lemma 2 (Correspondence of costs): For any policy $\pi \in$ $\Pi$ and any trajectory $\omega, c^{[d]}(\omega)=c_{d}(\omega)$.

Proof: Given a policy $\pi$, for any trajectory $\omega$, the cost cumulated up to time $d$ is the same for both the original and the surrogate problem. After time $d$, both $c_{d}(\omega)$ and $c^{[d]}(\omega)$ do not cumulate any additional cost, then the claim follows.

The following theorem represents the main result of this section

Theorem 3 (Suboptimality bound): The surrogate problem approximates the original problem according to

$$
\begin{aligned}
& \min _{\pi} \operatorname{AVaR}_{\tau}\left(c^{[d]}(\omega)\right) \leq \min _{\pi} \operatorname{AVaR}_{\tau}(c(\omega)) \\
& \leq \min _{\pi} \operatorname{AVaR}_{\tau}\left(c^{[d]}(\omega)\right)+\frac{n \bar{K}}{1-\tau} \frac{(1-\gamma)^{\lfloor(d+1) / n\rfloor}}{\gamma} .
\end{aligned}
$$

Proof: The left inequality is proven by noticing that $\min _{\pi} \operatorname{AVaR}_{\tau}(c(\omega)) \geq \min _{\pi} \operatorname{AVaR}_{\tau}\left(c_{d}(\omega)\right)=$ $\min _{\pi} \operatorname{AVaR}_{\tau}\left(c^{[d]}(\omega)\right)$, where the equality follows from Lemma 2 .

We now prove the right inequality. For any $s \in \mathbb{R}$ and policy $\pi$, one has

$$
\begin{aligned}
& \mathbb{E}\left[(c(\omega)-s)^{+}\right]=\mathbb{E}\left[(c(\omega)-s)^{+} \mid t^{*}(\omega) \leq d\right] \mathbb{P}\left(t^{*}(\omega) \leq d\right) \\
& \quad+\mathbb{E}\left[(c(\omega)-s)^{+} \mid t^{*}(\omega)>d\right] \mathbb{P}\left(t^{*}(\omega)>d\right)
\end{aligned}
$$

Let $c_{l}(\omega):=\sum_{t=d+1}^{\infty} c\left(x_{t}(\omega), u_{t}(\omega)\right)$ be the tail cumulated cost, and, as before, $c_{d}(\omega):=\sum_{t=0}^{d} c\left(x_{t}(\omega), u_{t}(\omega)\right)$. Since the function $x \rightarrow x^{+}$is sub-additive, i.e., $(x+y)^{+} \leq$ $x^{+}+y^{+}$and the expectation operator preserves monotonicity, one obtains the inequality

$$
\begin{aligned}
& \mathbb{E}\left[(c(\omega)-s)^{+} \mid t^{*}(\omega)>d\right] \\
& \quad=\mathbb{E}\left[\left(c_{d}(\omega)+c_{l}(\omega)-s\right)^{+} \mid t^{*}(\omega)>d\right] \\
& \quad \leq \mathbb{E}\left[\left(c_{d}(\omega)-s\right)^{+} \mid t^{*}(\omega)>d\right]+\mathbb{E}\left[c_{l}(\omega) \mid t^{*}(\omega)>d\right] .
\end{aligned}
$$

Furthermore, for each trajectory in the event set $\left\{\omega: t^{*}(\omega) \leq\right.$ $d\}$, one has

$\mathbb{E}\left[(c(\omega)-s)^{+} \mid t^{*}(\omega) \leq d\right]=\mathbb{E}\left[\left(c_{d}(\omega)-s\right)^{+} \mid t^{*}(\omega) \leq d\right]$

Collecting the results so far, one has the following inequalities:

$$
\begin{aligned}
& \mathbb{E}\left[(c(\omega)-s)^{+}\right] \\
& \leq \mathbb{E}\left[\left(c_{d}(\omega)-s\right)^{+} \mid t^{*}(\omega) \leq d\right] \mathbb{P}\left(t^{*}(\omega) \leq d\right) \\
& \quad+\mathbb{E}\left[\left(c_{d}(\omega)-s\right)^{+} \mid t^{*}(\omega)>d\right] \mathbb{P}\left(t^{*}(\omega)>d\right)+ \\
& \quad+\mathbb{E}\left[c_{l}(\omega) \mid t^{*}(\omega)>d\right] \mathbb{P}\left(t^{*}(\omega)>d\right) \\
& =\mathbb{E}\left[\left(c_{d}(\omega)-s\right)^{+}\right]+\mathbb{E}\left[c_{l}(\omega) \mid t^{*}(\omega)>d\right] \mathbb{P}\left(t^{*}(\omega)>d\right) \\
& \leq \mathbb{E}\left[\left(c_{d}(\omega)-s\right)^{+}\right]+\mathbb{E}\left[c_{l}(\omega)\right] .
\end{aligned}
$$

Equation (4) implies

$$
\begin{aligned}
& \min _{\pi} \operatorname{AVaR}_{\tau}(c(\omega)) \\
= & \min _{\pi} \min _{s \in \mathbb{R}}\left\{s+\frac{1}{1-\tau} \mathbb{E}\left[(c(\omega)-s)^{+}\right]\right\} \\
\leq & \min _{\pi} \min _{s \in \mathbb{R}}\left\{s+\frac{1}{1-\tau}\left(\mathbb{E}\left[\left(c_{d}(\omega)-s\right)^{+}\right]+\mathbb{E}\left[c_{l}(\omega)\right]\right)\right\} \\
\leq & \min _{\pi} \min _{s \in \mathbb{R}}\left\{s+\frac{1}{1-\tau}\left(\mathbb{E}\left[\left(c_{d}(\omega)-s\right)^{+}\right]\right)\right\} \\
& +\max _{\pi} \frac{1}{1-\tau} \mathbb{E}\left[c_{l}(\omega)\right] \\
= & \min _{\pi} \min _{s \in \mathbb{R}}\left\{s+\frac{1}{1-\tau}\left(\mathbb{E}\left[\left(c^{[d]}(\omega)-s\right)^{+}\right]\right)\right\} \\
& +\max _{\pi} \frac{1}{1-\tau} \mathbb{E}\left[c_{l}(\omega)\right] \\
= & \min _{\pi} \operatorname{AVaR}_{\tau}\left(c^{[d]}(\omega)\right)+\max _{\pi} \frac{1}{1-\tau} \mathbb{E}\left[c_{l}(\omega)\right],
\end{aligned}
$$

where the second to last equality follows from Lemma 2. We are left with the task of upper bounding $\mathbb{E}\left[c_{l}(\omega)\right]$. To this purpose, one can write

$$
\mathbb{E}\left[c_{l}(\omega)\right] \leq \bar{K} \sum_{t=d+1}^{\infty} \operatorname{Pr}_{\beta}^{\pi}\left(x_{t} \neq x_{M}\right) .
$$

Note that the result in Lemma 1 implies

$$
\begin{gathered}
\sum_{t=d+1}^{\infty} \operatorname{Pr}_{\beta}^{\pi}\left[x_{t} \neq x_{M}\right] \leq \sum_{k=\lfloor(d+1) / n\rfloor}^{\infty} \sum_{t=k n}^{(k+1) n-1} \operatorname{Pr}_{\beta}^{\pi}\left[x_{t} \neq x_{M}\right] \\
\leq \sum_{k=\lfloor(d+1) / n\rfloor}^{\infty} n(1-\gamma)^{k}=n \frac{(1-\gamma)^{\lfloor(d+1) / n\rfloor}}{\gamma} .
\end{gathered}
$$

The claim then follows immediately, as the above upper bound is policy-independent.

Note that according to Theorem 3 , as $d \rightarrow \infty$, the optimal cost of the surrogate problem recovers the optimal cost of the original problem, i.e., the surrogate problem provides a consistent approximation to the original problem, with a sub optimality factor that is computable from problem data.

Lemma 2 and Theorem 3 ensure that $c^{[d]}(\omega)$ can approximate $c(\omega)$ with arbitrary precision for a sufficiently large value of $d$. In the next section we then show how to solve the minimization problem:

$$
\min _{\pi} \operatorname{AVaR}_{\tau}\left(c^{[d]}(\omega)\right) .
$$

\section{Solution Algorithm}

Leveraging the surrogate problem from the previous section, we can now adapt the results proposed in [13] to solve problem (2). An essential step to solve this optimization problem is to compute $\mathbb{E}\left[\left(c^{[d]}(\omega)-s\right)^{+}\right]$, which entails deriving the probability distribution for the possible costs generated by the random variable $c^{[d]}(\omega)$. This problem can be solved by suitably augmenting the state space as described in the following, and then using occupancy measures. In the space of occupancy measures, an optimal policy is determined through the solution of a bilinear program, as 
explained below. For a given policy $\pi$ and initial distribution $\beta$, we define the occupancy measure for $(x, u) \in \mathcal{K}$ as

$$
\left.\rho(x, u)=\sum_{t=0}^{\infty} \operatorname{Pr}_{\beta}^{\pi}\left[x_{t}(\omega)=x, u_{t}(\omega)=u\right)\right] .
$$

Note that $\rho(x, u)$ is non negative but is in general not a probability itself. In the following we will use occupancy measures to determine the probability distribution of the total costs $c^{[d]}(\omega)$ and then to compute the needed expectation. According to the definition, occupancy measures depend on the policy $\pi$ and the initial distribution $\beta$. Given an absorbing MDP $\mathcal{M}=(X, U, \operatorname{Pr}, c)$, we define a new state-augmented absorbing MDP with additional state components that track the cumulated total cost and current stage. Although the original MDP $\mathcal{M}$ is finite and absorbing, the set of costs $c^{[d]}(\omega)$ generated by all possible policies can be very large, and this can subsequently lead to a linear program with an unmanageable number of decision variables. To counter this problem, we introduce a discretized approximation for $c^{[d]}(\pi, \beta)$ whose error can be arbitrarily bounded. To this end, we set $\zeta=\min \left\{\underline{K}, \frac{d \bar{K}}{N^{\prime}}\right\}$, where $N^{\prime} \in \mathbb{N}$ is a parameter describing the desired number of discretized values for the cumulated cost. Due to Assumption 1, $\zeta$ is strictly positive. The effective number of different values is $N=\left\lceil\frac{d \bar{K}}{\zeta}\right\rceil$. This value may be higher than $N^{\prime}$ due to our definition of $\zeta$. We then define a new MDP $\mathcal{M}_{N}^{\prime}=\left(X^{\prime}, U^{\prime}, \operatorname{Pr}^{\prime}, c^{\prime}\right)$ as follows. Its state space is $X^{\prime}=X \times \mathbb{N}_{N} \times \mathbb{N}_{d}$, where $\mathbb{N}_{N}=\{0,1, \ldots, N\}$ and $\mathbb{N}_{d}=\{0,1, \ldots, d\}$. Elements in the augmented states will be indicated as $(x, y, z)$. As clarified in the following, the two additional components store the cumulated running cost $(y)$ and current stage $(z)$. Recall that in the surrogate problem, after $d$ steps, the state is guaranteed to have entered the absorbing set, i.e., it is guaranteed that $x_{d}(\omega)=x_{M}$. Thus the value of the $z$ component is in $\mathbb{N}_{d}=\{0,1, \ldots, d\}$. On the other hand the input sets are defined as $U^{\prime}(x, y, z)=U(x) . X^{\prime}$ and $U^{\prime}$ induce a new set $\mathcal{K}^{\prime}=\{(x, y, z, u) \mid(x, u) \in \mathcal{K} \wedge y \in$ $\left.\mathbb{N}_{N} \wedge z \in \mathbb{N}_{d}\right\}$. The new cost function $c^{\prime}: \mathcal{K}^{\prime} \rightarrow \mathbb{R}_{\geq 0}$ is $c^{\prime}(x, y, z, u)=c(x, u)$. The transition probability function is modified as follows:

$$
\begin{aligned}
& \operatorname{Pr}^{\prime}\left(\left(x^{\prime}, y^{\prime}, z^{\prime}\right) \mid(x, y, z), u\right)= \\
& \begin{cases}\operatorname{Pr}\left(x^{\prime} \mid x, u\right) & \text { if } y^{\prime}=y+\left\lfloor\frac{c(x, u)}{\zeta}\right\rfloor \wedge z^{\prime}=z+1 \wedge z^{\prime}<d \\
1 & \text { if }\left(x^{\prime}, y^{\prime}, z^{\prime}\right)=\left(x_{M}, y, d\right) \wedge z=d \\
0 & \text { otherwise }\end{cases}
\end{aligned}
$$

As evident from the definition of the new transition function, the new variables included in the state stores the discretized 3 running cost and the stage. Consistently with our definition of the surrogate problem, the revised transition function includes a timeout that imposes a transition to the absorbing state $x_{M}$ after $d$ steps, and from that point onwards the accrued cost does not change. Note also that the additional state components $y$ and $z$ are deterministic functions of the previous state and control input $u$. Extending the formerly introduced notation, for a given trajectory $\omega$ of $\mathcal{M}_{N}^{\prime}$, we write $y_{t}(\omega)$ for the second component of the state at time

\footnotetext{
${ }^{3}$ To be precise, the discretized running cost is scaled by $\zeta$.
}

$t$ and $z_{t}(\omega)$ for the third component. Finally, for a given initial distribution $\beta$ on $X$, we define the following new initial distribution $\beta^{\prime}$ on $X^{\prime}$,

$$
\beta^{\prime}(x, y, z)=\left\{\begin{array}{ll}
\beta(x) & \text { if } y=0 \wedge z=0 \\
0 & \text { otherwise }
\end{array} .\right.
$$

Note that the properties of $\mathcal{M}$ carry over to $\mathcal{M}_{N}^{\prime}$. In particular, if Assumptions 1, 2 hold for $\mathcal{M}$ then they hold for $\mathcal{M}_{N}^{\prime}$ too, and, if $\mathcal{M}$ is absorbing, then $\mathcal{M}_{N}^{\prime}$ is also absorbing. Thus we indicate with $X^{\prime T}$ its transient set of states. For a given realization $\omega$, consider now $c_{t}^{[d]}(\omega)=\sum_{i=0}^{t} c\left(x_{i}, u_{i}\right)$, i.e., the true cumulative cost of the surrogate MDP problem without discretization. The following theorem establishes that even though the approximation error introduced by discretizing the running cost grows linearly with $t$, it is possible to bound it with arbitrary precision.

Theorem 4: For each $\varepsilon>0$ and each $t \in\{0, \ldots, d\}$, there exists a discretization step $\zeta$ such that $\left|\zeta y_{t}(\omega)-c_{t}^{[d]}(\omega)\right|<\varepsilon$. Proof. Pick $\zeta=\varepsilon / d$. Let $e(t)=c_{t}^{[d]}(\omega)-\zeta y_{t}(\omega)$ be the approximation error at time $t$. Note that by definition $e(t) \geq 0$ and $e(0)=c_{0}^{[d]}(\omega)-\zeta y_{0}(\omega)=0$. From the definition of the transition probability function, $P^{\prime}$, it follows that $e(t+1) \leq e(t)+\zeta$, which implies $e(d) \leq d \zeta=\varepsilon$. Since for $t>d$ we have $e(t)=e(d)$, the claim follows.

A key step towards the solution of problem in (5) is therefore to derive the statistical description of the discretized total cost $y_{d}(\omega)$ that is used to approximate $c^{[d]}(\omega)$. This objective can be achieved by exploiting the occupancy measures for the state-augmented $\operatorname{MDP} \mathcal{M}^{\prime}$. For $(x, y, z, u) \in \mathcal{K}^{\prime}$, the occupancy measure on $\mathcal{M}^{\prime}$ induced by a policy $\pi$ and an initial distribution $\beta$ is given as:

$$
\begin{aligned}
& \rho(x, y, z, u)= \\
& \sum_{t=0}^{\infty} \operatorname{Pr}_{\beta}^{\pi}\left[x_{t}(\omega)=x, y_{t}(\omega)=y, z_{t}(\omega)=z, u_{t}(\omega)=u\right]
\end{aligned}
$$

The occupancy measure, $\rho$, is a vector in $\mathbb{R}_{\geq 0}^{\left|\mathcal{K}^{\prime}\right|}$, i.e., it is a vector with $\left|K^{\prime}\right|$ non negative components. The set of legitimate occupancy vectors is constrained by the initial distribution $\beta$ and defined by the policy $\pi$. It is well known [1] that these constraints can be expressed as follows:

$$
\begin{gathered}
\sum_{\left(x^{\prime}, y^{\prime}, z^{\prime}\right) \in X^{\prime T}} \sum_{u \in A\left(x^{\prime}, y^{\prime}, z^{\prime}\right)} \rho\left(x^{\prime}, y^{\prime}, z^{\prime}, u\right)\left[\delta_{(x, y, z)}\left(x^{\prime}, y^{\prime}, z^{\prime}\right)-\right. \\
\left.P^{\prime}\left(\left(x^{\prime}, y^{\prime}, z^{\prime}\right) \mid(x, y, z), u\right)\right]=\beta(x, y, z) \forall(x, y, z) \in X^{\prime T}
\end{gathered}
$$

where $\delta_{x}(y)=1$ if and only if $y=x$. For $0 \leq k \leq N$ we introduce random variables $\theta(k)$ with the property that $\theta(k)=\operatorname{Pr}\left[y_{d}(\omega)=k\right]$. This is easily achieved using occupancy measures, i.e., $\theta(k)=\sum_{(x, y, z, u) \in \mathcal{K}^{\prime}} I(y=k \wedge$ $z=d) \rho(x, y, z, u)$, where $I(\cdot)$ is the indicator function equal to 1 when its argument is true, and 0 otherwise. Note that by definition $\theta(k)$ is equal to $\operatorname{Pr}\left[y_{d}(\omega)=k\right]$, and by Theorem $4 y_{d}(\omega)$ approximates $c^{[d]}(\omega)$ with arbitrary precision. Combining the above definitions we then get to the 
following problem whose solution approximates the solution to (5):

$$
\min _{\rho, \theta} \min _{s \in[0, \bar{K} d]} s+\frac{1}{1-\tau} \sum_{y \in \mathbb{N}_{N}}(y-s)^{+} \theta(y)
$$

s.t.

$$
\begin{gathered}
\sum_{\left(x^{\prime}, y^{\prime}, z^{\prime}\right) \in X^{\prime T}} \sum_{u \in A\left(x^{\prime}, y^{\prime}, z^{\prime}\right)} \rho\left(x^{\prime}, y^{\prime}, z^{\prime}, u\right)\left[\delta_{(x, y, z)}\left(x^{\prime}, y^{\prime}, z^{\prime}\right)-\right. \\
\left.P^{\prime}\left(\left(x^{\prime}, y^{\prime}, z^{\prime}\right) \mid(x, y, z), u\right)\right]=\beta(x, y, z) \forall(x, y, z) \in X^{\prime T} \\
\theta(k)=\sum_{(x, y, z, u) \in \mathcal{K}^{\prime}} I(y=k \wedge z=d) \rho(x, y, z, u), 0 \leq k \leq N .
\end{gathered}
$$

When comparing this last optimization problem with (5), the reader will note that the variable $s$ is constrained in the interval $[0, \bar{K} d]$. Indeed, the objective function is continuous with respect to $s$, and it is straightforward to verify that the partial derivative of the objective function with respect to $s$ is negative for $s<0$ and positive for $s>\bar{K} d$. The objective function given in Eq. (7) is concave with respect to $\theta(y)$ and is defined over a convex feasibility set [13]. To the best of our knowledge, there exist no efficient methods to determine the global minimum for this class of problems. Hence, the problem is approximately solved fixing different values of $s$ within the range $[0, \bar{K} d]$, and then solving the corresponding linear problem over the optimization variables $\rho$ and $\theta$. Comparing the problem in Eq. (7) with the one in Eq. (2) one might initially think that the objective function in Eq. (2) does not depend on the policy $\pi$. However, the dependency on $\pi$ is carried over by the occupancy measure $\rho$, as evident from Eq. (6). Moreover, it is well known from the theory of constrained MDPs [1] that there is a one to one correspondence between policies and occupancy measures, i.e., every policy defines a unique occupancy measure and every occupancy measure induces a policy.

\section{NUMERICAL EXPERIMENTS}

To illustrate the performance of the proposed algorithm, we adopt the rapid deployment scenario considered in [6], [8]. A graph is used to abstract and model the connectivity of a given map of an environment (see, e.g., [14]). One robot is positioned at a start vertex and is tasked to reach the goal vertex within a given temporal deadline while providing some guarantee about its probability of successfully completing the task. When moving from vertex to vertex, the robot can choose from a set of actions, each trading off speed with probability of success. In particular, actions with rapid transitions between two vertices have higher probability of failure; and conversely when the robot moves slowly between two vertices it has a higher probability of success. In this scenario, failure means that the robot does not move (e.g., fails to pass through an opening), so elapsed time increases without making progress towards the goal. With a given temporal deadline $T$ and success probability $P$, the robot is tasked to reach the target vertex "safely" (such that the true mission success probability is at least $P$ ), while satisfying the temporal constraint. From a design perspective it is of interest to know if there exists a policy $\pi$ achieving this objective, and to compute it. If the policy does not exist, it is of interest to know how to modify the parameters in order to make the task feasible.
In our previous work we solved this problem by modeling it using Constrained Markov Decision Processes (CMDP). In the CMDP approach, one maximizes the probability of success while imposing a constraint on the temporal deadline. However, this method only returns risk-neutral policies, i.e., the resultant policies only guarantee that the temporal deadline is met in expectation, and there is no explicit control on the tail probability of the constraint. As a radical departure from the original problem formulation, the AVaR minimization method proposed in Eq. (2) searches for a policy that is feasible with respect to the temporal deadline constrain $4^{4}$ and systematically controls the worstcase variability of total travel time. Note that a policy with low success probability will have large tail probability in total travel time even if the expected temporal deadline is met. Therefore the optimal policies from AVaR minimization will have high success probability. This motivates the application of AVaR minimization to rapid robotic deployment. First, note that, in the devised setting, the robot will eventually reach the final goal with positive probability. However, due to possible failures one cannot put an a-priori bound on the random total travel time. Therefore, the total cost criterion is indeed a natural choice for this task. Moreover, Assumptions 1 and 2 are easily justified because the immediate cost function (i.e., time to move) is always positive and the global reachability property follows from the graph structure.

To illustrate the performance of risk-averse deployment, two different policies are compared. Here both policies are computed using unconstrained stochastic control methodologies for which the immediate cost is the travel time between two vertices, and the actions correspond to all possible node transitions on the graph. The first is the classic risk-neutral policy obtained with value iteration. The second is a risk averse policy obtained with the algorithm presented in this paper using $\tau=0.95$. For each policy, 1000 executions are run, and the distribution of total travel time is reported. Figures 2 and 3 show the distributions for the two cases. The risk-neutral policy obtains a lower expected cost, but has a longer tail, as evidenced by the 61 instances with a cost larger than or equal to 15 (notice that $T=15$ is the desired time of completion in this example). Moreover, as evidenced by the shape of the histogram, costs are more spread out. The risk averse policy, on the other hand, results in less variability as desired. Less than 30 instances have a cost larger or equal than 15 , a reduction of the weight of the tail by more than one half. Importantly, when computing a risk-neutral policy using classic methods like policy iteration or value iteration, one is merely provided with a policy that minimizes the expected cost (in our case time to completion), and no additional information is readily available. With our approach instead, one not only obtains a policy minimizing the AVaR criterion, but a statistical description of the costs is also obtained as a byproduct. That is to say, for each discretized completion time $k$, the probability $\operatorname{Pr}\left[c^{[d]}=k\right]$ is computed as well, thus unveiling the relationship between the time to complete the deployment task and its probability. This is shown in Figure 4 for different values of $\tau$. Hence, if

\footnotetext{
${ }^{4}$ For any random variable $Z$ with finite expectation, $\operatorname{AVaR}_{\tau}(Z) \geq \mathbb{E}[Z]$ for $\tau \in[0,1]$. Therefore, if the solution to the AVaR minimization problem is bounded above by the temporal deadline, then the corresponding minimizer is also a feasible policy to the original problem.
} 


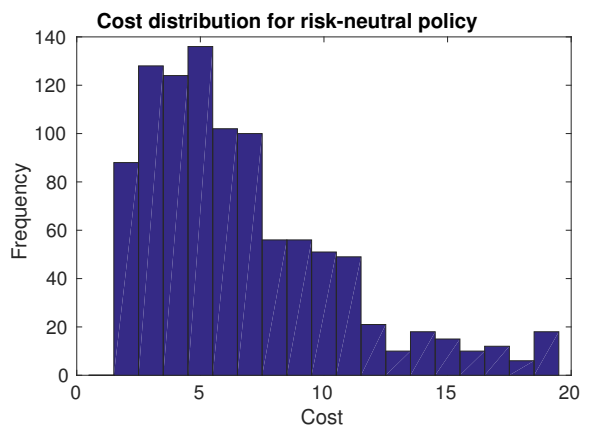

Fig. 2: Cost distribution for a risk-neutral policy

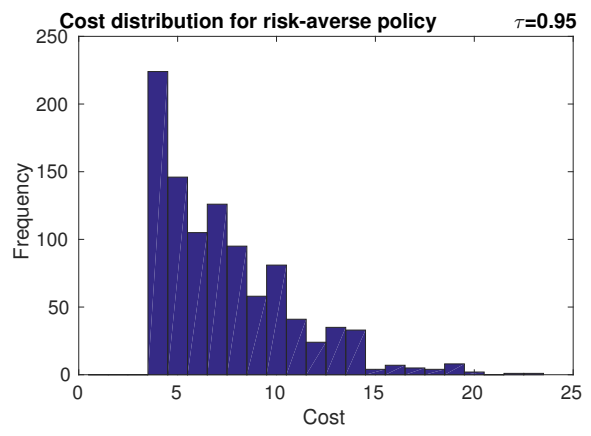

Fig. 3: Cost distribution for a risk averse policy with $\tau=$ 0.95 .

the computed policy does not meet the desired performance, the designer has information on how to tune $T$ and $P$.

\section{CONCLUSIONS}

In this paper we have considered how risk aversion in MDPs can be introduced jointly with the AVaR risk metric under the total cost criterion. Our results advance the state of the art because AVaR has only been previously considered in MDPs with finite horizon or discounted infinite horizon cost criteria. Such extension is important as the total cost criterion appears as a natural model for robotic applications, and is non-straightforward as current algorithms, e.g., from [13] and [3], only work with bounded cumulated costs (which is not the case for total cost formulations). Under two mild assumptions, an approximation algorithm with provable sub-optimality gap was provided. Furthermore, a

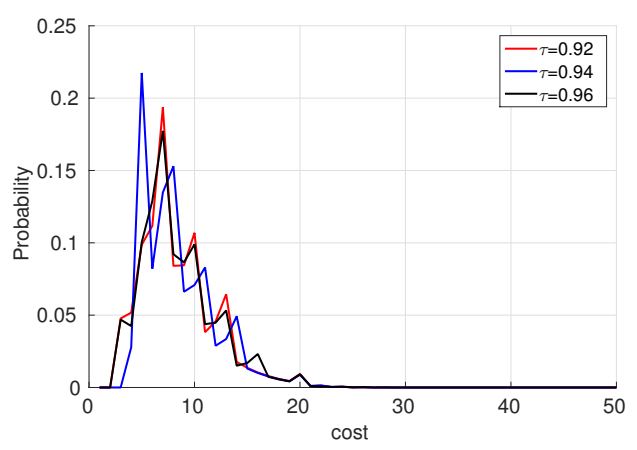

Fig. 4: Comparison of probability cost distribution for different values of $\tau$. rapid deployment scenario was used to demonstrate that riskaversion gives more informative policies when compared to traditional risk-neutral formulations. While our findings focus on risk averse MDPs with an AVaR risk metric, our approach can be easily extended along multiple dimensions. In particular, by exploiting the results presented in [13], it is possible to use our approximation for a broader range of risk metrics, i.e., metrics that are uniformly continuous and law invariant. Moreover, since the algorithm we considered is based on occupancy measures, it can be easily extended to the CMDP case. This will be the focus of future work.

\section{REFERENCES}

[1] E. Altman. Constrained Markov Decision Processes. Stochastic modeling. Chapman \& Hall/CRC, 1999.

[2] P. Artzner, F. Delbaen, J. Eber, and D. Heath. Coherent Measures of Risk. Mathematical Finance, 9(3):203-228, 1999.

[3] N. Bäurle and J. Ott. Markov Decision Processes with Average-Valueat-Risk Criteria. Mathematical Methods of Operations Research, 74(3):361-379, 2011

[4] D. Bertsekas. Dynamic Programming \& Optimal Control, volume 1 \& 2. Athena Scientific, 2005.

[5] V. Borkar and R. Jain. Risk-constrained Markov Decision Processes. IEEE Transaction of Automatic Control, 59(9):2574 - 2579, 2014.

[6] S. Carpin, M. Pavone, and B. Sadler. Rapid Multirobot Deployment with Time Constraints. In Proc. of the IEEE/RSJ Int. Conf. on Intelligent Robots and Systems, pages 1147-1154, 2014.

[7] Y. Chow and M. Ghavamzadeh. Algorithms for CVaR Optimization in MDPs. In NIPS 27, pages 3509-3517, 2014.

[8] Y. Chow, M. Pavone, B. Sadler, and S. Carpin. Trading Safety Versus Performance: Rapid Deployment of Robotic Swarms with Robus Performance Constraints. ASME Journal of Dynamical Systems, Measurements and Control, 137(3):031005, 2015.

[9] E. Feinberg. Handbook of Markov Decision Processes: Methods and Applications. Springer, 2012.

[10] J. Filar, D. Krass, and K. Ross. Percentile Performance Criteria for Limiting Average Markov Decision Processes. IEEE Transaction of Automatic Control, 40(1):2-10, 1995.

[11] R. Gallager. Stochastic Processes: Theory for Applications. Cambridge University Press, 2013.

[12] R. Howard and J. Matheson. Risk-sensitive Markov Decision Processes. Management Science, 18(7):356-369, 1972.

[13] W. Haskell and R. Jain. A Convex Analytic Approach to Riskaware Markov Decision Processes. SIAM Journal of Control and Optimization, 2014

[14] A. Kolling and S. Carpin. Extracting Surveillance Graphs from Robot Maps. Proc. of the IEEE/RSJ Int. Conf. on Intelligent Robots and Systems, 2323-2328, 2008.

[15] S. Mannor and J. Tsitsiklis. Algorithmic Aspects of Mean-variance Optimization in Markov Decision Processes. European journal of Operational Research, 231:645-653, 2013.

[16] L. Prashanth. Policy Gradients for CVaR-constrained MDPs. In Algorithmic Learning Theory, pages 155-169. Springer, 2014.

[17] R. Rockafeller and S. Uryasev. Optimization of Conditional Value at Risk. Journal of Risk, 2:21-41, 2000.

[18] R. Rockafeller and S. Uryasev. Conditional Value-at-risk for General Loss Distributions. Journal of Banking Finance, 26:1443-1471, 2002.

[19] G. Serraino and S. Uryasev. Conditional Value-at-risk (CVaR). In Encyclopedia of Operations Research and Management Science, pages 258-266. Springer, 2013.

[20] D. P. Bertsekas and J. N. Tsitsiklis. An Analysis of Stochastic Shortest Path Problems. In Mathematics of Op. Res., 16(3):580-595, 1991.

[21] H. Yu and D. P. Bertsekas. Q-learning and policy iteration algorithms for stochastic shortest path problems. In Annals of Operations Research, 208(1):95-132, 2012.

[22] E. A. Feinberg and J. Huang. On the Reduction of Total-Cost and Average-Cost MDPs to Discounted MDPs. In Technical Report, available at arXiv:1507.00664. 2015.

[23] Y. Chow and A. Tamar and S. Mannor and M. Pavone. Risk-Sensitive and Robust Decision-Making: a CVaR Optimization Approach. In Advances in Neural Information Processing Systems, 2015.

[24] M. Sobel. The Variance of Discounted Markov Decision Processes. Journal of Applied Probability, pages 794-802, 1982.

[25] A. Tamar, Y. Glassner, and S. Mannor. Optimizing the CVaR via Sampling. In AAAI, 2015.

[26] S. Thrun, W. Burgard, and D. Fox. Probabilistic Robotics. MIT Press, 2006 